\title{
Accuracy of children's and parents' memory for a novel painful experience
}

\author{
Melanie A Badali BAHons, Rebecca R Pillai BAHons, Kenneth D Craig PhD, Kelly Giesbrecht BSc, Christine T Chambers MA
}

\author{
MA Badali, RR Pillai, KD Craig, K Giesbrecht, CT Chambers. \\ Accuracy of children's and parents' memory for a novel pain- \\ ful experience. \\ Pain Res Manage 2000;4(2):161-168.
}

BACKGROUND: Despite recent progress in understanding memory of pain in adults, the validity of the assumption that these findings extend to children has not been established. Because treatment often is evaluated on the basis of pain recall, it is crucial that the accuracy of pain memories in children be established.

OBJECTIVES: To examine children's ability to recall pain intensity and contextual details associated with a novel painful experience. Furthermore, children's memories were compared with those of their parents.

SUBJECTS AND METHODS: Twenty-three parent-child dyads were recruited from a sample participating in an earlier study investigating children's responses to the cold pressor test and parents' characterization of the children's responses. Children (age five to 12 years) and parents independently had rated the child's pain using a seven-point Faces Pain Scale. Approximately one year later, they were asked to recall the experience and rate the pain again. Memory for contextual details associated with the event also was assessed through a series of open-ended questions.

RESULTS: A 2 (rater) $\times 2$ (time period) repeated measures ANOVA examined the reliability of ratings and differences between parent and child ratings of pain, both recorded at the time of the cold pressor and recalled one year later. There were no significant differences in ratings over time or between parent and child. CONCLUSIONS: The findings indicate that children's memories of a novel painful experience can be consistent over a long interval. Moreover, parents' ratings also reflect good recall and can be in agreement with those of their children.

Key Words: Children; Intensity; Memory; Pain; Parents; Selfreport

\section{Fidélité du souvenir d'une nouvelle expérience douloureuse chez des enfants et leurs parents}

CONTEXTE : Malgré les progrès récents réalisés dans la compréhension du souvenir d'un événement douloureux chez les adultes, on n'a pas encore établi la validité de l'assertion selon laquelle les résultats obtenus s'appliquent également aux enfants. Comme l'évaluation des traitements repose souvent sur le souvenir de la douleur, il est crucial de déterminer la fidélité des souvenirs d'expériences douloureuses chez les enfants.

OBJECTIFS : Vérifier la capacité des enfants à se rappeler l'intensité de la douleur et les détails contextuels associés à une nouvelle expérience douloureuse; comparer les souvenirs des enfants à ceux de leurs parents.

SUJETS ET MÉTHODE : Vingt-trois paires parents-enfants ont été recrutées à partir d'un échantillon de sujets ayant déjà participé à une étude sur la réaction des enfants à une épreuve au froid et la caractérisation de cette réaction par les parents. Les enfants (de 5 à 12 ans) et les parents ont coté séparément la réaction à la douleur à l'aide de l'échelle Faces Pain Scale à sept niveaux. Environ un an plus tard, on leur a demandé de se remémorer l'expérience et de coter de nouveau la douleur. Le rappel des détails associés à l'événement a aussi été évalué à l'aide d'une série de questions ouvertes.

RÉSULTATS : On a procédé à des mesures répétées (deux évaluateurs, deux reprises) de l'analyse de la variance pour vérifier la fiabilité des cotes et les écarts d'évaluation de la douleur entre les parents et les enfants, et ce, tant au moment de l'expérience qu'un an plus tard. Aucune différence significative de cotes n'a été enregistrée dans le temps ainsi qu'entre les parents et les enfants.

CONCLUSIONS : Les résultats indiquent que le souvenir d'une nouvelle expérience douloureuse chez les enfants peut se conserver fidèlement sur une longue période. De plus, les cotes des parents font état d'un bon souvenir et elles peuvent concorder avec celles de leurs enfants. 
$U^{\prime}$ nderstanding pain and memory is a challenge to both researchers and clinicians because the phenomena are subjective by definition. When the focus of inquiry is a child, the tasks of defining and documenting memory for pain, and eliciting the child's unique perspective is even more difficult (1). Pain has been described as subjective (2), and a child's experience of pain likely involves somatosensory elements, affective components and contextual information, all of which are subject to interpretation and distortion over time (3). A rich, highly personal and salient experience such as this is likely to be represented in memory, yet little is known about children's capacity to remember pain. This gap in the knowledge base needs to be narrowed, given that studies of children's memory for painful experiences are relevant to clinical practice, as is our understanding of pain perception, cognitive development and memory representation (3).

In a recent review by Ornstein et al (3), 14 studies concerning children's memory for pain and painful events were cited. Since that time, one further study (4) has been added to the growing body of literature. However, very few of these studies have explicitly investigated memory for the sensory component of pain associated with an event (5-7). Moreover, the existing results are not congruent.

The first studies to address the topic of memory for children's pain explored the consistency among reports of remembered events. Lehmann et al (5) found that children (aged three to eight years) did not consistently evaluate the relative difference in pain intensity of recalled painful events, using picture, block-based and triad scales (5) on two occasions one week apart. In contrast, Bieri et al (8) demonstrated that six-year-old children's ratings of a recent recalled painful experience using the Faces Pain Scale were relatively consistent over a two-week interval. Bruck et al (9) advanced the field by providing independent, objective confirmation of a painful event in their examination of the influence of postevent suggestions on children's reports of their visits to a pediatrician. Children's ratings on the 'How Much Did It Hurt Scale' (9) one week after the painful event were positively correlated with distress ratings made by research assistants at the time of the inoculation. Approximately one year after the inoculation, children who did not receive any suggestive feedback made ratings of hurt that were significantly lower than their ratings one week following the shot. In essence, all the studies discussed so far examined the consistency or reliability of memory for pain but did not address the issue of the accuracy of the memories compared with pain actually reported at the time of the experience.

Lander et al (6) addressed this limitation by examining the relationships among expected, experienced and recalled pain in response to a venepuncture by using a $100 \mathrm{~mm}$ vertical Visual Analogue Scale (VAS). Children between five and 17 years of age had a $43 \%$ accurate recall of their pain intensity $( \pm 9 \mathrm{~mm})$ after approximately two months. In contrast, Zonneveld et al (7) observed a considerably higher level of accuracy in their study of inpatients' (aged five to 16 years) recall of pain intensity. Using the Faces Pain Scale (8), children's recalled worst pain intensities were $80 \%$ accurate af- ter one day and on average $72.4 \%$ accurate after one week. These results were more similar to the accuracy of recalling remembered pain using the Faces Pain Scale (8).

Discrepancies across studies may occur because of different methodologies, including pain measures (eg, faces scales versus VAS), participant demographics (eg, ages three to eight years versus five to 16 years), inpatient versus research participant, pain events (eg, inoculation versus daily pains), and delay between pain and memory assessments (eg, one week versus one year). Furthermore, the interpretation of findings of memory for clinical pains can be confounded by variables inherent in the clinical setting, such as the meaning of the pain, procedure or illness to the child, the status of the child's illness, technical difficulties in performing a medical procedure, anxiety levels of parents and children, and analgesic medication (10).

It is imperative that concerted attempts be made to overcome the difficulties inherent in this research area in order to increase our knowledge of pain memory (11). Pain memories play a major role in clinical practice. First, complaints of pain, present or past, are the main stimulus for seeking professional aid and advice (12). Furthermore, clinicians use patients' recall of pain and its attributes as an important source of evidence in assessing, diagnosing and choosing treatment for pain, as well as for evaluating improvement following treatment. Frequently, pain reports given by children, including their memory of pain, are discredited in research or clinical settings (6). In other cases, children are unable to verbally report their pain because of their developmental level, emotional distress or verbal limitations (13). Consequently, parents often are relied upon to provide proxy reports of pain for children (14). Further research is needed to determine the accuracy of children's memory for pain and compare it with caregivers' memories of the experience.

The present study sought to expand the base of knowledge concerning memory for pain by investigating children's and parents' memory for pain intensity and contextual details associated with a novel painful experience. The cold pressor test was used to induce pain, allowing rigorous control over the stimulus (eg, duration, repetition) as well as the situation in which it was presented. See LeBaron et al (10) for a review of the advantages, feasibility and usefulness of the cold pressor pain paradigm in children, and Chen et al (15) for a discussion of the cold pressor tonic pain model and its generality for clinical pain and human pain responsivity. The use of the cold pressor test provided a unique opportunity to examine memory over a long delay while reducing the influence of previously acquired knowledge on memory for the pain stimulus. Children and parents rated the child's pain intensity during and approximately one year after the cold pressor test.

In summary, the purpose of this study was to investigate the accuracy of children's recall of their pain intensity, examine the accuracy of parent's recall of the intensity of their child's pain experience, and investigate children's and parents' memories of contextual details associated with the painful experience. 


\section{Participants}

\section{SUBJECTS AND METHODS}

The sample comprised 23 children (nine females and 14 males, mean age 7.57 years, SD 1.90) and one of their parents (13 mothers and seven fathers; mean age 40.16 years, SD 5.61) who had participated in a study examining developmental differences in the congruence of multiple measures of pain in children (16). Participants were recruited through flyers that invited them to telephone the Pain Research Laboratory at the University of British Columbia if they were interested in participating in a follow-up study. A total of 114 parent-child dyads participated in the initial study. Seventythree flyers were mailed to families who agreed to be contacted by mail, and nine families were not contacted due to administrative constraints (eg, change of address). Inclusion criteria were age four to 12 years, English speaking, no known cognitive disability, parent's consent to participate, child's assent to participate and no prior experience of the cold pressor test (except in the initial study [16]). Three parents had more than one child participate, so data from the second child tested (and the responses from the parent concerning this child) were excluded to satisfy the assumption of independence necessary for the statistics performed. The final sample included in the analysis consisted of 20 children (eight females and 12 males, mean age 7.6 years, SD 1.88). Children ranged from four to 11 years of age, with four children aged four to five years, 10 children aged six to eight years and three children aged nine to 11 years.

\section{Materials}

Cold pressor apparatus: Pain stimulation was produced with the cold pressor apparatus, a safe and widely used painful stimulus that meets the criteria important for experimental noxious stimulation (eg, stimulus controllability, reliability, discriminability, convenience and validity) (17). The apparatus used a commercially manufactured plastic cooler measuring $23.5 \mathrm{~cm}$ wide, $43.5 \mathrm{~cm}$ long and $28.0 \mathrm{~cm}$ deep with an $11 \times 11 \mathrm{~cm}$ square opening in the top. The water was maintained at a constant temperature of $11^{\circ} \mathrm{C}\left( \pm 1^{\circ}\right)$.

Rating scale: Pain intensity was measured with the Faces Pain Scale (8), which consists of seven diagrammatic faces. Empirical evidence indicates that child's sex and age minimally influence reliability of the Faces Pain Scale (8) and that it is a valid choice of faces scales due to its neutral 'no pain' face (18). A preliminary investigation for ratio scale properties of this scale (8) indicates that parametric statistics may be used. The scale was initially administered with the following instructions:

Each of these faces is of a person who has no hurt or pain, or some, or a lot of hurt or pain. The first face doesn't have any hurt or pain at all. Do you see that face? Okay, The next face has just a little bit of hurt or pain. Each of the next faces has a little more hurt or pain, until you get to this last face, who has the worst hurt or pain you can imagine.
Children and parents were asked to provide independent ratings of the child's pain after the first $10 \mathrm{~s}$ of the cold pressor test, after $30 \mathrm{~s}$ and immediately before hand removal. For the purposes of the present study, the highest pain score reported during the cold pressor test was operationalized as an estimate of the most pain experienced. At follow-up, the first seven letters of the alphabet were placed in a random order under the Faces Pain Scale (specifically the letters F, D, A, G, $\mathrm{C}, \mathrm{B}$ and $\mathrm{E}$ were placed from left to right under the faces) to facilitate its use over the telephone. Letters were chosen so as not to introduce a numerical scale into the faces scale. Reinstruction in the use of the Faces Pain Scale included an orientation to the letters underneath the faces. After each question, parents and children were instructed to "please choose the face that shows how much hurt or pain you would have" and "please tell me the name of the letter directly under that face."

\section{Procedure}

The study was approved by the University of British Columbia's Behavioural Research Ethics Board. Preliminary data collection occurred as a part of a larger study conducted by Chambers and colleagues (16). The initial phase of the study took place at Science World, a museum featuring scientific activities and displays geared toward children, located in Vancouver, British Columbia. Demographic information and participant ratings of pain associated with the cold pressor test were obtained at that time. In addition, children were asked to rate how much hurt or pain they would have if they got a big needle at the doctor's office or got stung by a bee. The follow-up phase occurred approximately one year after the cold pressor test was administered. A telephone followup was chosen because some of the participants resided outside of Vancouver, and a similar methodology had been used successfully in a previous study examining memory of pain (6).

Participants who contacted the laboratory received general information about the nature of the study from a trained research assistant, and a telephone interview was arranged if they were interested in participating. Follow-up interviews occurred anywhere from 295 days to 341 days (mean 310 days, SD 12.29 days) after initial testing. At the beginning of the interview, verbal consent was obtained, and parents were asked to continue the study out of earshot of their children. After being reinstructed in the use of the Faces Pain Scale, parents were asked to use the scale to answer the question, "What do you think is the most hurt or pain your child felt during the cold pressor test?" Finally, a structured interview protocol was administered to assess recall of defined features of the cold pressor test experience. Open-ended questions probed for recall for time of year, new people and behavioural pain tolerance, as well as events before, during and after the cold pressor test (see Table 1 for a list of questions). Answers to the questions were predefined. Participants were judged to be correct if they provided an answer within the parameters of the definition. However, if a correct response was accompanied by a feature that was false, the answer was judged to be incorrect. Two coders judged the accu- 
TABLE 1

Children's and parents' recall of contextual details

\begin{tabular}{|c|c|c|}
\hline \multirow[b]{2}{*}{ Questions (parent modifications) } & \multicolumn{2}{|c|}{ Correct answers (\%) } \\
\hline & Parent & Child \\
\hline $\begin{array}{l}\text { 1. During what season did you (and your } \\
\text { child) participate in the study at Science } \\
\text { World? }\end{array}$ & 35 & 30 \\
\hline $\begin{array}{l}\text { 2. During what month did you (and your } \\
\text { child) participate in the study at Science } \\
\text { World? }\end{array}$ & 30 & 15 \\
\hline $\begin{array}{l}\text { 3. When you (your child) had his/her hand in } \\
\text { the cold water, how many researchers } \\
\text { were with you? }\end{array}$ & 80 & 65 \\
\hline 4. How many researchers were female? & 80 & 55 \\
\hline $\begin{array}{l}\text { 5. Where was the strap or band we put } \\
\text { around you (your child) that counted the } \\
\text { number of times your (his/her) heart beat } \\
\text { in one minute? }\end{array}$ & 60 & 20 \\
\hline $\begin{array}{l}\text { 6. What colour was the cold pressor (clarified } \\
\text { with "the thing holding the water")? }\end{array}$ & 60 & 95 \\
\hline $\begin{array}{l}\text { 7. Where was your mom or dad sitting while } \\
\text { you had your hand in the cold water? } \\
\text { (Where were you sitting, in relation to your } \\
\text { child, while he/she had her hand in the } \\
\text { cold water?) }\end{array}$ & 65 & 50 \\
\hline $\begin{array}{l}\text { 8. What colour hair did the girl who sat } \\
\text { beside you (your child) during the cold } \\
\text { pressor have? }\end{array}$ & 85 & 70 \\
\hline $\begin{array}{l}\text { 9. What did you (your child) dry her/his hands } \\
\text { with after the cold pressor? }\end{array}$ & 55 & 80 \\
\hline $\begin{array}{l}\text { 10. What did you (your child) receive after the } \\
\text { study? }\end{array}$ & 55 & 70 \\
\hline $\begin{array}{l}\text { 11. Did you (your child) leave her/his hand in } \\
\text { the water until the timer beeped? }\end{array}$ & 80 & 75 \\
\hline
\end{tabular}

racy of classification of responses, and disagreements were resolved through discussion.

When parents had answered all the experimenter's questions, they were asked to bring their child to the telephone. The experimenter then repeated the same protocol with the child. At the end of the study, parents and children were debriefed and remunerated $\$ 20$ for their participation.

\section{Design}

A 2 (rater) $\times 2$ (time of rating) within-subjects design with pain intensity ratings as the dependent variable was employed. Factor one, time of recall, had two levels - rating during the painful event versus recall approximately one year after the painful stimulus was administered. Factor two, rater, had two levels - recording of pain intensity by child versus recording of pain intensity by parent. This permitted determination of whether recalled pain intensity ratings were significantly different after a delay of one year for either or both the parent and child. Examination of the interaction between these two factors allowed the authors to ascertain whether children's initial ratings of pain in response to the cold pressor test and their recall of pain intensity differed significantly from parents' ratings of the pain intensity experi-
TABLE 2

Two-way repeated measures ANOVA means and standard deviations of worst pain intensity as measured by the Faces Pain Scale

\begin{tabular}{|c|c|c|c|}
\hline \multicolumn{2}{|c|}{ Reported pain intensity* (initial) } & \multicolumn{2}{|c|}{ Reported pain intensity* (recall) } \\
\hline Parent $^{\dagger}$ & Child $^{+}$ & Parent $^{\dagger}$ & Child $^{+}$ \\
\hline $1.9 \pm 1.21$ & $2.45 \pm 2.04$ & $2.35 \pm 1.31$ & $2.25 \pm 1.71$ \\
\hline
\end{tabular}

enced by their child. Pearson's correlations and Cohen's kappas were also used to assess the accuracy of memory for pain.

For descriptive purposes, associated pain accuracy ratings were computed by subtracting recalled pain intensity ratings from ratings made during the cold pressor test. Accuracy scores for contextual details were computed by calculating the percentage of questions answered correctly.

\section{RESULTS}

\section{Children's and parents' recall of associated pain}

A 2 (rater) $\times 2$ (time of rating) repeated measures ANOVA was conducted to analyze the Faces Pain Scale scores reported by parents and children both during the pain event and at the one year follow-up (Table 2). The main effects were not of interest in this analysis (ie, the main effects of rater collapsed over time and of time collapsed over rater); thus, only results pertaining to the interaction are reported. The interaction of time and rater was found to be nonsignificant, indicating no differences among the four mean pain intensity ratings (parent during, child during, parent recall, child recall) $(\mathrm{F}[1,19]=2.74, \mathrm{P}>0.05)$. A post hoc power analysis indicated that the sample size was sufficient to detect a moderate effect size had it been present, with a 0.82 probability of correctly retaining a true null hypothesis (19). This result suggests that the lack of a significant difference was not due to the small sample analyzed. In sum, children's and parents' recalled pain intensity ratings do not differ significantly from their own reports at the time of the pain stimulus. Furthermore, regardless of the time of rating, parent and child pain intensity scores were not significantly different.

The strength of the relationship between pain intensity scores given at the time of the pain event and at follow-up were analyzed. Pearson correlations were computed for the two parent pain intensity scores and the two child pain intensity scores. The resulting correlations for the parents $(\mathrm{r}=0.52$, $\mathrm{P}<0.05)$ and for the children $(\mathrm{r}=0.63, \mathrm{P}<0.005)$ were found to be significant. Insufficient power precluded subsequent tests to explore whether there was a significant difference between the two correlations.

To examine the possibility that the similarity of initial and recalled pain ratings was due more to stable individual differences in use of rating scales than to memory, ratings of pain associated with hypothetical pain experiences (not remembered experiences) collected during the initial phase of the study were used as covariates in the analysis of the correlation between children's initial and recalled pain ratings. In ef- 
fect, this placed everybody on the same scale during the initial phase (ie, controlling for differences in the way children use the scale). The partial correlation of children's initial pain intensity ratings with recalled pain intensity ratings, partialling out the hypothetical pain intensity related to a bee sting during the initial phase was $\mathrm{pr}=0.63, \mathrm{P}<0.01$. The partial correlation of children's initial pain intensity ratings with recalled pain intensity ratings, partialling out the hypothetical pain intensity related to a needle during the initial phase was $\mathrm{pr}=0.59, \mathrm{P}<0.01$. Because the partial correlations were significant, these results suggest that the stability from the initial phase to follow-up was not due solely to stable individual differences in the way children use the pain scale.

Cohen's kappa statistic was used to assess the accuracy of memory (20). Rather than using kappa as a measure of specific accuracy (ie, ability to select the same face on two occasions), kappa was used as a measure of clinically relevant accuracy. Children's and parents' pain ratings were collapsed into two groups - a no pain/low pain group (faces 0 to 2 on the Faces Pain Scale) and a clinically significant pain group (faces 3 to 6 on the Faces Pain Scale) for each of the two rating periods (initial assessment and recall assessment) (Table 3). This classification, which is based on the cutoff for clinically significant pain established by Gauthier et al (21), has been used by previous researchers investigating agreement between reports of pain (22). Kappa statistics for agreement between initial and recalled ratings of pain for no pain/low pain versus clinically significant pain represented fair to good agreement beyond chance for both children $(=0.48, \mathrm{P}<0.05)$ and parents $(=0.48, \mathrm{P}<0.05)(23-25)$.

Although, overall, both parents and children were found to be fairly reliable, patterns of inaccuracies that were present were described. Accuracy scores for the Faces Pain Scale were calculated in the same way as by Zonneveld et al (7) to facilitate comparison. Accuracy scores for most pain intensity were computed by subtracting the maximum of the experienced most pain intensity from the recalled most pain intensity. As per Zonneveld et al (7), the resulting difference scores were classified as underestimates (the recalled pain intensity less than the experienced pain intensity by more than one face), accurate estimates (the difference between recalled pain intensity and experienced pain was less than or equal to plus or minus one face) and overestimates (the recalled pain intensity was greater than the experienced pain intensity score by more than one face). Sixty-five per cent of children were accurate, $15 \%$ underestimated their most pain intensity and $20 \%$ overestimated their most pain intensity. Eighty-five per cent of parents were accurate, and $15 \%$ overestimated their most pain intensity ratings. Unfortunately, there was insufficient power to test properly whether the accuracy of children's recalled pain intensities were different from those of their parents by using these classifications.

\section{Children's and parents' recall of contextual details}

Questions were asked to determine the recall ability of both parents and children for the contextual details related to the pain event. For each group (parents and children), the con-
TABLE 3

Percentage of children and parents rating pain in either the no pain/low pain group or the clinically significant pain group at the time of the initial pain assessment and at the time of recall

\begin{tabular}{llcc}
\hline & & \multicolumn{2}{c}{ Recall ratings } \\
Rater & Initial ratings & $\begin{array}{c}\text { No/low } \\
\text { pain* }\end{array}$ & $\begin{array}{c}\text { Clinically } \\
\text { significant pain }\end{array}$ \\
\hline Child $(n=20)$ & $\begin{array}{l}\text { No/low pain* } \\
\text { Clinically significant } \\
\text { pain }\end{array}$ & $50(10)$ & $20(4)$ \\
& $5(1)$ & $25(5)$ \\
Parent $(n=20)$ & $\begin{array}{l}\text { No/low pain* } \\
\text { Clinically significant } \\
\text { pain }^{\dagger}\end{array}$ & $50(10)$ & $5(1)$ \\
& $20(4)$ & $25(5)$ \\
\hline
\end{tabular}

*Faces 0 to 2 on the Faces Pain Scale; ${ }^{\dagger}$ Faces 3 to 6 on the Faces Pain Scale. Numbers in parentheses are the numbers of participants.

textual pain accuracy score was defined as the mean percentage of correctly recalled answers. The contextual pain accuracy scores were $57.27 \%$ (SD $18.21 \%$ ) and $65.91 \%$ (SD $18.15 \%$ ) for children and parents, respectively. A $t$ test indicated that parents and children were not differentially accurate at recalling contextual details of the pain experience $(\mathrm{t}[19]=1.44, \mathrm{P}>0.05)$. The percentage of children and parents who were able to remember correctly each of several predefined details associated with the pain event can be seen in Table 1 .

\section{DISCUSSION}

The results from three complementary sets of analyses examining different parameters of agreement between initial and recalled pain ratings (ie, repeated measures ANOVA, Pearson's correlations, kappa statistics) converged to suggest that children's memory for pain is fairly reliable and accurate. This conclusion is congruent with that of other studies on memory for pain in children using the Faces Pain Scale $(7,8)$. Furthermore, the modest correlations obtained in the present study were similar in magnitude to those calculated in previous studies of adult memory for pain (11). In an attempt to address Morley's criticism (26) that correlations do not provide an assessment of accuracy and that one cannot use group means on self-report measures as anything other than proxy measures of accuracy, kappa statistics were also used. Kappa statistics indicated fair to good agreement above chance between initial and recalled pain for children when their ratings were collapsed into two groups (ie, a no pain/low pain group and a clinically significant pain group). The results of the present study diverge from those found in other studies $(5,6,9)$, which indicated lower accuracy of children's memory for pain intensity. However, such comparisons are tenuous due to methodological differences between studies.

The lower accuracy of recall for pain intensity found by Lehmann et al (5) and Bruck et al (9) may be due in part to the use of pain intensity measures of questionable reliability and validity (27). For example, the inconsistent responses to the multidimensional pain scales used by Lehmann et al (5) may reflect a problem with self-report rather than recall. The mul- 
tiplicity of cues in Lehmann et al's study (5) may have confused their young subjects $(27)$. Indeed, studies $(7,8)$ that used more established instruments (eg, Faces Pain Scale) found higher accuracy for pain memory. Even among established instruments, differences were reported. For example, Lander et al (6) found lower accuracy for recall of pain intensity using a VAS than did the present study, and Zonneveld et al (7), who used the Faces Pain Scale. Bruck et al (9) found that children showed significant reductions in their ratings on the How Much Did It Hurt Scale (9), an adaptation of the Poker Chip Tool (28) in the year following their shot. This tentatively suggests that the children were not reliable. However, Bruck et al (9) did not provide any data regarding the percentage of children who accurately recalled their rating. It is imperative that future research is conducted with psychometrically reliable and valid age appropriate measures.

The discrepancy between accuracy of children's recall of pain intensities found in the present study and others may reflect the different samples studied. One basic factor that can affect the strength and organization of memory representations is the age of the individual $(3,29)$. The positive relationship between age and accuracy of recalled pain intensity is consistently found across studies $(5,7,30)$. The sample in the present study was not large enough to permit analyses of the relationship between age and recall.

The comparatively higher accuracy found by the present study may be due, in part, to the marked differences in stimulus events being remembered across studies. Painful events previously investigated range from rather common events such as venepuncture (6) to more unusual procedures such as the voiding cystourethrogram $(30,31)$. The painful events differ in terms of many characteristics, which need to be taken into account when interpreting results. For example, novel events may be more likely to be recalled than more routine events $(32,33)$. The use of a novel pain stimulus in the present study may account for the higher accuracy found in this study compared with other studies that measured memory for non-novel stimuli $(5,6)$.

Use of a novel pain stimulus in the present study minimized the interpretive problem that arises when considering children's recall of events that have been experienced on multiple occasions (3). Previously, Lander et al (6) attempted to control for interference by similar experiences by excluding participants who had experienced other injections or venepuncture in the period between the pain experience and the memory assessment. However, this can only be considered a partial control because it does not account for the influence of prior experience. Prior knowledge of events to be remembered can influence encoding, storage and subsequent retrieval of information (34-38). For example, a child who has a considerable amount of experience with injections may have formed generalized representations or scripts for that experience (39). A child's script can influence the way the experience is encoded, stored and/or recalled (3). Prior knowledge of painful procedures such as venepuncture may serve to distract the child from making accurate ratings, leading them to draw instead upon scripted information stored in memory. In the present study, the novel pain experience of the cold pressor test is not likely to be confounded by prior knowledge or experience.

Another issue that complicated comparability of results was the delay between pain ratings. The literature reports memory assessments from one day (7) to one year (9) after the initial pain ratings. Furthermore, the time baseline measurements that were collected vary across studies (eg, during or immediately after the painful stimulus [6] versus one week after the initial pain experience [9]) and up to four years after the event (40). It has been hypothesized that the impact of scripts may increase as the delay between initial recording and recall increases (3). As time passes between the pain experience and baseline or memory assessment, children may have more difficulty remembering the particular incident and, instead, may report information congruent with their scripts. Although scripts can facilitate understanding of experiences, they may also lead to distortion in remembering any specific instance (3). This may partially explain why Zonneveld et al (7) found that children's accuracy for remembering average versus worst pain was not different after one day, but there was a greater decrement in memory accuracy for worst pain after a time lag of seven days in participants who only recalled pain once. It is possible that individuals' use of scripts enhanced their ability to remember their average pain compared with their worst pain, or that the use of scripts interfered with memory for worst pain. Thus, the present study may have showed relatively high accuracy after a longer delay than other studies $(6,7)$ because the design was unconfounded by the impact of experience.

The present study was unique in that parents' ratings were also unlikely to be affected by specific scripts as the cold pressor test was novel for all participants. The majority of parents accurately recalled children's pain intensity after approximately one year. Furthermore, parents and children did not significantly differ in their accuracy levels. This was demonstrated by both repeated measures analyses and kappa statistics. No known prospective studies have been conducted comparing children's memory for pain intensity with their parents' ratings. Chambers et al (22) have shown that many parents fail to identify clinically significant pain in their children. Even if parents' reports of their child's clinical or everyday pains are highly reliable, and their memories are accurate, this does not mean that their initial ratings were adequate interpretations of the child's subjective state. Because children (aged four to 12) also appear to be able to provide information on memory for pain approximately as reliably and potentially more accurately than their parents, information from both children and caregivers should be integrated when assessing pain and planning treatment.

In general, children accurately recalled answers to the majority of the questions asked about contextual details of the pain event at follow-up. These findings are in congruence with research on children's event memory, which has documented children's ability to remember the details of relatively unusual experiences over months (41) and years (42). Events linked to a specific time and place appear to be more 
readily recalled (39). Parents also accurately recalled answers to the majority of the questions asked about contextual details of the pain event. Overall, parents and children recalled contextual details associated with the cold pressor test to a similar extent. This finding is in line with previous research indicating that mothers and children (aged eight to 11 years old) tended similarly to recall physical features of daily injury events such as the location of child and of caregiver, time of day and type of treatment received (33).

Although the present data have implications for understanding children's and parents' memories of pain, the evidence reported here can only be offered tentatively. First, the small sample size prevents comment on any age-related changes in accuracy, and the results may easily be influenced by the age distribution of the sample. Moreover, the sample was not randomly selected, and the unique group of parents and children studied (eg, people who visited Science World and agreed to participate in two phases of the study) may not be representative of the general population. Second, although the cold pressor test serves as an analogue to clinical pain, the findings reflect recall of the pain stimulus used, but they do not provide direct information on memory for clinical pain. Unlike many cases of clinical pain, the cold pressor test was not administered repeatedly over time. Although this prevented the applicability of the present findings to pain events that occur repeatedly over long periods of time (eg, headache, recurrent abdominal pain), this had the advantage of ensuring that previous knowledge was unlikely to influence recall performance. Finally, pain is a complex experience

\section{REFERENCES}

1. Steward MS, O'Connor J. Pediatric pain, trauma, and memory. Curr Opin Pediatr 1994;6:411-7.

2. Bonica JJ. The need of a taxonomy. Pain 1979;6:247-52.

3. Ornstein PA, Manning EL, Pelphrey KA. Children's memory for pain. J Dev Behav Pediatr 1999;20:262-77.

4. Koutantji M, Pearce SA, Oakley DA, Feinmann C. Children in pain: An investigation of selective memory for pain and psychological adjustment. Pain 1999;81:237-44.

5. Lehmann HP, Bendebba M, DeAngelis C. The consistency of young children's assessment of remembered painful events. J Dev Behav Pediatr 1990;11:128-34.

6. Lander J, Hodgins M, Fowler-Kerry S. Children's pain predictions and memories. Behav Res Ther 1992;30:117-24.

7. Zonneveld LNL, McGrath PJ, Reid GJ, Sorbi MJ. Accuracy of children's pain memories. Pain 1997;71:297-302.

8. Bieri D, Reeve RA, Champion GD, Addicoat L, Ziegler JB. The Faces Pain Scale for the self-assessment of the severity of pain experienced by children: Development, initial validation, and preliminary investigation for ratio scales properties. Pain 1990;41:139-50.

9. Bruck M, Ceci SJ, Francoeur E, Barr R. "I hardly cried when I got my shot": Influencing children's reports about a visit to their pediatrician. Child Dev 1995;66:193-208.

10. LeBaron SA, Zeltzer L, Fanurik D. An investigation of cold pressor pain in children (part I). Pain 1989;37:161-71.

11. Erskine A, Morley S, Pearce S. Memory for pain: A review. Pain 1990;41:255-65.

12. Hunter M, Philips C, Rachman S. Memory for pain. Pain 1979;6:35-46

13. Chambers CT, McGrath PJ. Pain measurement in children. In: Ashburn MA, Rice LJ, eds. The Management of Pain. New York: Churchill Livingstone, 1998:625-34. that has both sensory and affective components. The study would have benefited from inclusion of a measure of the affective domain of pain. These limitations should encourage researchers to continue to explore the impact of pain, in laboratory as well as naturalistic settings, on memory.

Despite the limitations of the present study, the findings suggest that, under certain conditions, children are capable of providing consistent reports of painful events that they have experienced and that the accuracy of their reports do not differ significantly from those of their parents. The present findings add to the growing body of literature on children's memory for pain by investigating both pain intensity and contextual details associated with pain, using a standardized novel pain stimulus and comparing children's memories with those of their parents. Further prospective research is needed to build upon the findings of this exploratory study and link the research in this area to current models of memory.

ACKNOWLEDGMENTS: Preparation of this article was supported by a British Columbia Health Research Foundation Studentship to MA Badali, a University of British Columbia Graduate Fellowship to RR Pillai, a Social Sciences and Humanities Research Council of Canada grant to KD Craig and a Medical Research Council of Canada Doctoral Award to CT Chambers. We gratefully acknowledge the participants and the staff of Science World for their cooperation with this project. We also sincerely thank Dr Carl von Baeyer for his extensive contributions to the revision of this article.

14. McGrath PJ, Finley GA, Ritchie J. Parents' roles in pain assessment and management. Int Assoc Study Pain Newsl 1994:3-4.

15. Chen ACN, Dworkin SF, Haug J, Gehrig J. Human pain responsivity in a tonic pain model: Psychological determinants. Pain 1989;37:143-60.

16. Chambers CT, Cszisar A, Craig KD, McGrath PJ, Pillai, RR. Developmental differences in the congruence of multiple measures of pain in children. Pain Res Manage 2000;5(Suppl A):32A-3A. (Abst)

17. Hirsch MS, Liebert RM. The physical and psychological experience of pain: The effects of labeling and cold pressor temperature on three pain measures in college women. Pain 1998;77:41-8.

18. Chambers CT, Giesbrecht K, Craig KD, Bennett SM, Huntsman E. A comparison of faces scales for the measurement of pediatric pain: children's and parents' ratings. Pain 1999;83:25-35.

19. Cohen J. Statistical Power Analyses for the Behavioural Sciences. New York: Academic Press, 1977.

20. Cohen J. A coefficient of agreement for nominal scales. Educ Psychol Meas 1960;20:37-46.

21. Gauthier JC, Finley GA, McGrath PJ. Children's self-report of postoperative pain intensity and adequacy of medication: establishing a link. Clin J Pain 1998;14:116-20.

22. Chambers CT, Reid GJ, Craig KD, McGrath PJ, Finley GA. Agreement between child and parent reports of pain. Clin J Pain 1998; $14: 336-42$.

23. Dworkin SF, Whitney CW. Relying on objective and subjective measures of chronic pain: guidelines for use and interpretation. In: Turk DC, Melzack R, eds. Handbook of Pain Assessment. New York: Guilford Press, 1992:429-46.

24. Fleiss J. Statistical methods for rates and proportions. New York: John Wiley and Sons, 1981. 
25. Landis JR, Koch GG. The measurement of observer agreement for categorical data. Biometrics 1977;33:159-74.

26. Morley SJ. Reliability and accuracy of memory for pain. Pain 1994;57:132.

27. Erickson CJ. Pain measurement in children: Problems and directions. J Dev Behav Pediatr 1990;11:135-7.

28. Hester NO, Foster RL, Kristensen K. Measurement of pain in children: Generalizability and validity of the pain ladder and the poker chip tool. In: Tyler DC, Krane EJ, eds. Advances in Pain Research and Therapy, vol 15. New York: Raven Press, 1979:79-84.

29. Nelson K. Event Knowledge. Hillsdale: Lawrence Erlbaum, 1986.

30. Merritt KA, Ornstein PA, Spicker B. Children's memory for a salient medical procedure: Implications for testimony. Pediatrics 1994;94:17-23.

31. Goodman GS, Quas JA, Batterman-Faunce JM, Riddlesberger MM, Kuhn J. Children's reactions to and memory for a stressful event: Influences of age, anatomical dolls, knowledge, and parental attachment. Appl Dev Sci 1997;1:54-75.

32. Hudson JA. Memories are made of this: General event knowledge and development of autobiographic memory. In: Nelson K, ed. Event Knowledge. Hillsdale: Lawrence Erlbaum, 1986:97-118.

33. Peterson L, Harbeck C, Moreno A. Measures of children's injuries: Self-reported versus maternal-reported events with temporally proximal versus delayed reporting. J Pediatr Psychol 1993;18:133-47.

34. Bjorklund FF. The role of conceptual knowledge in the development of organization in children's memory. In: Brainerd CJ, Pressley M, eds. Basic Processes in Memory Development. New York: Springer-Verlag, 1985:103-42.

35. Chi MTH, Ceci SJ. Content knowledge: Its role representation and restructuring in memory development. In: Reese HW, ed. Advances in Child Development and Behavior, vol 20. Orlando: Academic Press, 1987:91-142.

36. Clubb PA, Nida RE, Merritt KA, Ornstein PA. Visiting the doctor: Children's knowledge and memory. Cognit Dev 1993;8:361-72.

37. Ornstein PA, Larus DM, Clubb PA. Understanding children's testimony: Implications of research on the development of memory. In: Vasta R, ed. Annals of Child Development, vol 8. London: Jessica Kingsley, 1991:145-76.

38. Ornstein PA, Naus MJ. Effects of the knowledge based on children's memory strategies. In: Reese HW, ed. Advances in Child Development and Behavior, vol 19. Orlando: Academic Press, 1985:113-48.

39. Fivush R. Learning about school: The development of kindergartners' school scripts. Child Dev 1984;55:1697-709.

40. Beyer JE, Berde CB, Bournaki MC. Memories of pain in three to seven-year old children. J Pain Symptom Manage 1991;6:174.

41. Fivush R, Hudson J, Nelson K. Children's long-term memory for a novel event: An exploratory study. Merrill Palmer Q 1984;30:303-16.

42. Hudson JA, Fivush R. As time goes by: Sixth graders remember a kindergarten experience. Appl Cognit Psychol 1991;5:347-60. 


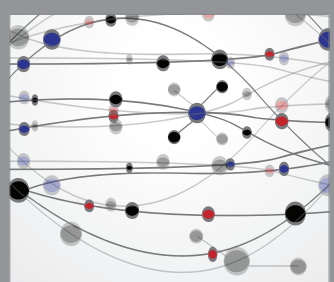

The Scientific World Journal
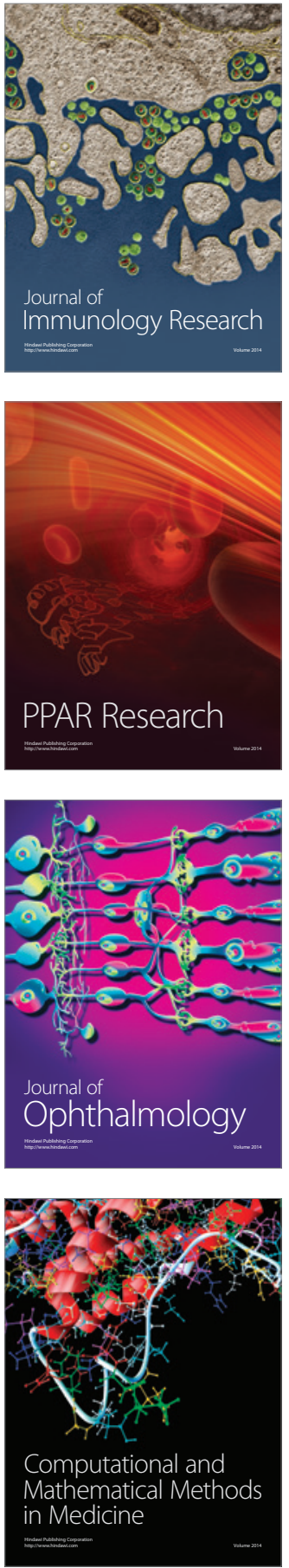

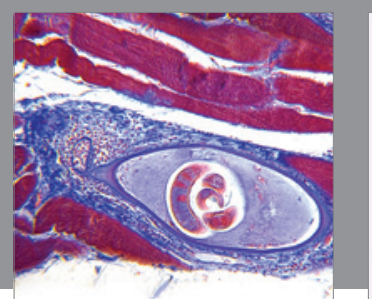

Gastroenterology Research and Practice

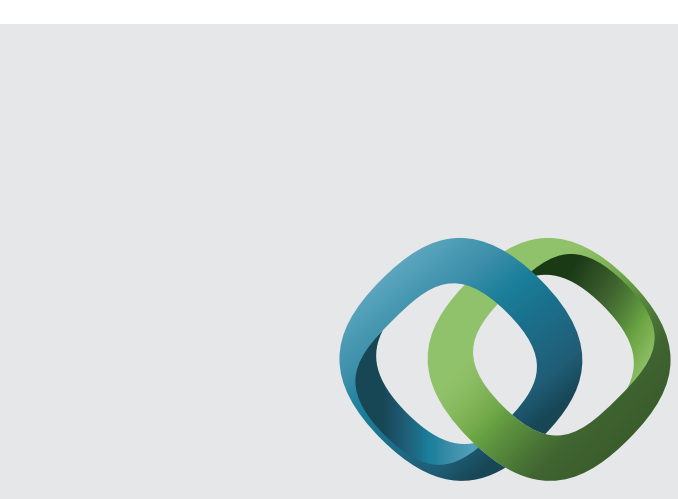

\section{Hindawi}

Submit your manuscripts at

http://www.hindawi.com
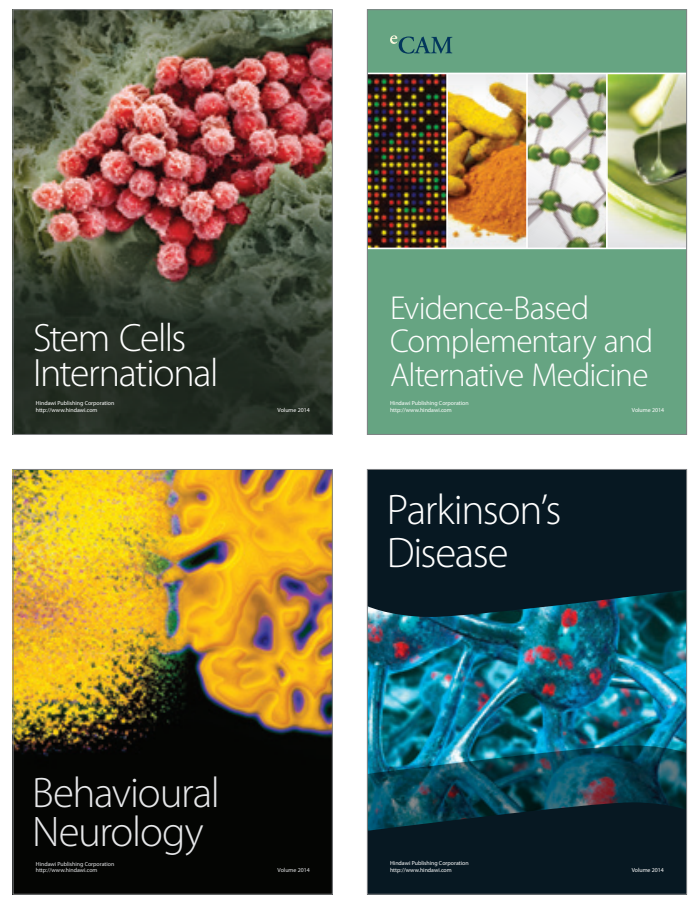
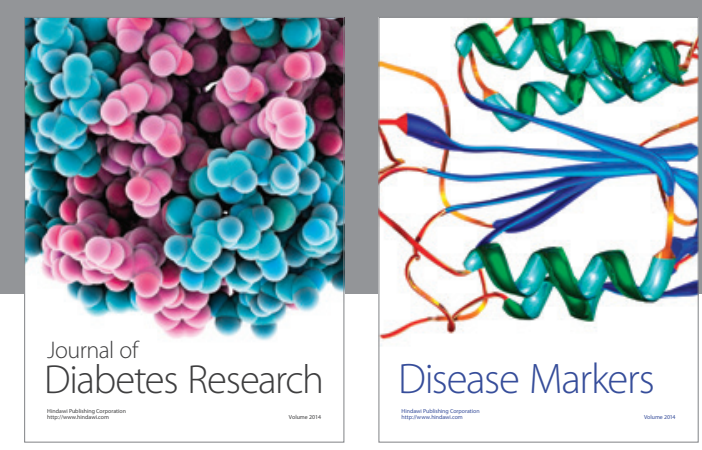

Disease Markers
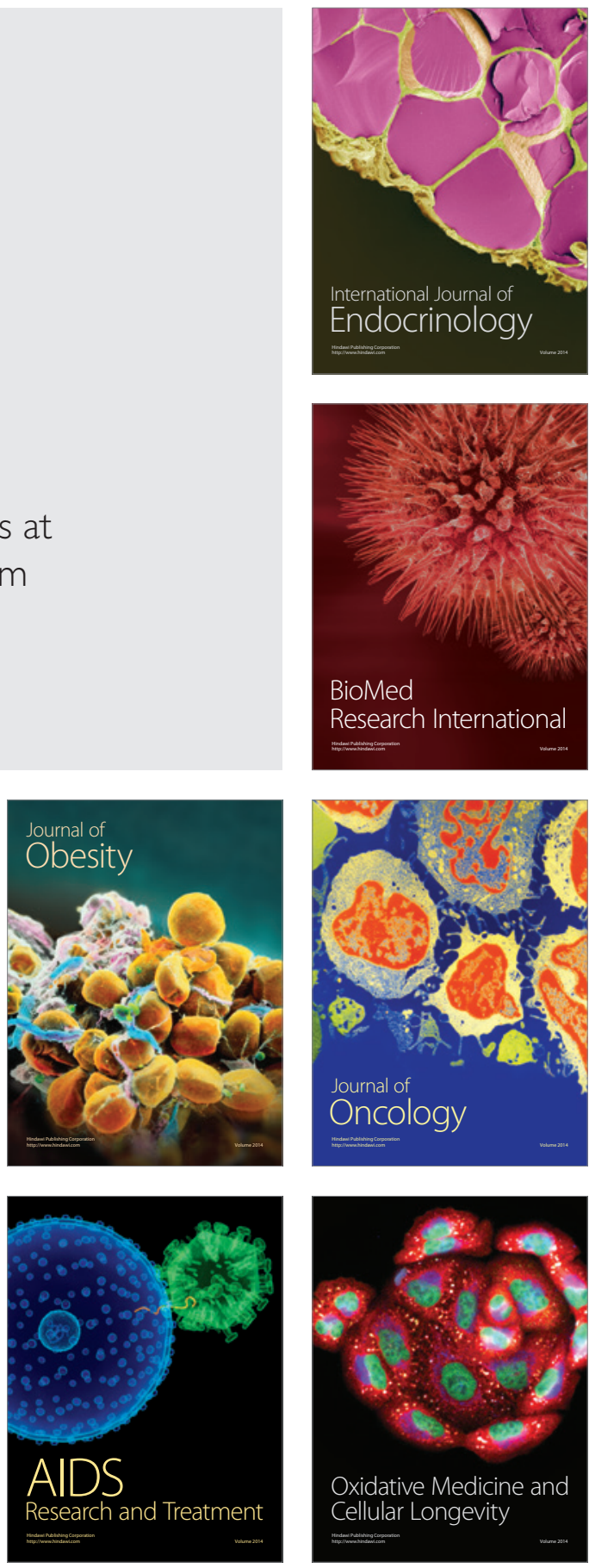